\title{
Deployment of wireless sensor network in dispersed renewable energy sources for increasing efficiency of power distribution networks
}

\author{
Theodoros A. TSIFTSIS ${ }^{1}$, Paschalis C. SOFOTASIOS ${ }^{2}$, \\ Nick PAPANIKOLAOU ${ }^{3}$, Michael I. LOUPIS ${ }^{1}$
}

\begin{abstract}
In this work, a novel performance analysis method for evaluating the robustness of emerging power distribution networks (PDNs), which involve deployable renewable energy sources, is proposed. This is realized with the aid of the outage probability (OP) criterion in the context of cooperative communications, which is widely considered in modern wireless communication systems. The main usefulness of this method is that it allows the involved components to communicate to each-other by means of a robust and flexible wireless sensor network architecture. In this context, any conventional medium voltage (MV) bus of the PDN is represented as a wireless relay node where data signals gathered from each MV bus can be forwarded reliably to a control station for the subsequent processing. The received signals at wireless nodes are decoded and then forwarded to ensure minimal errors and maximal robustness at the receiving site. The considered OP analysis denotes the probability that the power of a received information signal drops below a pre-defined threshold which satisfies the acceptable Quality of Service requirements of a reliable signal reception. To this end, simple closed-form expressions are proposed for the OP of
\end{abstract}

CrossCheck date: 4 February 2015

Received: 24 May 2014/Accepted: 19 January 2015/Published online: 2 June 2015

(C) The Author(s) 2015. This article is published with open access at Springerlink.com

$\triangle$ Theodoros A. TSIFTSIS

tsiftsis@teiste.gr

1 Technological Educational Institute (T.E.I.) of Central Greece, 35100 Lamia, Greece

2 Tampere University of Technology, 33101 Tampere, Finland

3 Democritus University of Thrace, Kimmeria, 67100 Xanthi, Greece a regenerative cooperative-based PDN in the presence of various multipath fading effects, which degrade information signals during wireless transmission. The offered results are rather simple and provide meaningful insights for the design and deployment of smart grid systems.

Keywords Smart grid, Cooperative communications (CCs), Power distribution network (PDN), Outage probability, Renewable energy source (RES), Wireless sensor network (WSN)

\section{Introduction}

Electric power generation systems constitute an invaluable part of human life. Based on this, power generation has been effectuated through different sources in order to meet the extensive demands of any geographical and populated area in an efficient and robust manner. To this effect and with the currently wide awareness for critical global ecological and environmental matters, the need for critical requirements and actions on the generation, transmission and consumption of energy resources has been raised extensively [1]. This has resulted in demands for more ecological sources of energy over conventional methods of production as well as more efficient management of resources that additionally secure indirect energy savings [2]. This trend has been also supported and promoted strongly by national and international political and societal authorities, which in turn have increased the attention of both industry and academia in providing effective solutions that can create a societal, environmental and economic impact [1-3].

Renewable energy sources (RESs) constitute an important alternative source of energy that is researched and exploited rapidly. A crucial task of RES based systems is 
their efficient and robust integration in the core power network and particularly when referring to microgrids. This is not a straightforward task due to the different characteristics, specifications and demands of each RES, which have been taken into account for ensuring smooth and efficient network operation. By additionally recalling the critical involvement of different technical domains such as power electronics, automation, control, sensors and actuators as well as power network analysis, the need for robust network performance and reliable service provision becomes more evident. More specifically, the centralized RES units typically infuse energy in the power networks of high and extra high voltage levels. As a consequence the produced energy experiences substantial losses related to transmission and distribution as well as economic costs due to the need for non-trivial infrastructure capital investment in non-dense rural and remote areas [4]. Recalling that the contribution of RES units in the overall power network is increasing and anticipating that the corresponding levels will be over $20 \%$, the aforementioned effects are undoubtedly non-negligible and must be addressed effectively.

Potential solutions have been proposed in the context of efficient and low-cost power electronic based conversion structures and driving methods [5-8] and the references therein. As a result, various efficient and reliable power electronic conversion solutions and effective driving techniques have been developed for the connection of RES units to the distribution level [7-9]. However, these converters appear to have an important operating problem as they are incapable of accounting for the current demands of power distribution networks (PDNs), which ultimately burdens adequate penetration of RES. This in turn increases the need for addressing satisfactorily the merge of different production units so as to ensure acceptable service coverage. This operation is critical and often challenging as certain units can contribute in the greater power networks partially and/or seasonally. Thus, adequate compensation methods must be taken into account which are typically realized by installing additional alternative power sources or, more importantly, employing storage devices and units. Those are rather useful in autonomous microgrid structures that typically establish connection to the greater PDN asynchronously [10].

The design and structure of the corresponding inverters must meet the specifications for serving the various power production sources simultaneously while they are required to cooperate with respective storing units so that a high quality and operation efficiency is ultimately feasible [1113]. Evidently the reliable and robust operation of power networks, which involve various RES in different geographical locations, requires accurate quantification and continuous control of numerous factors and characteristics both in regular and in transient conditions. Notably, it is particularly important accounting for such conditions due to critical voltage disturbances by short circuits, coupling/ decoupling processes of large loads and resonance by nonlinearity. Typical methods to overcome such destructive effects include, among others, the use of active filters (AFs), dynamic voltage restorers (DVRs), multistage capacitors and conventional voltage support elements such as transformers with on load tap chargers [11-17] and the references therein. As a result, it is undoubtedly important to guarantee accurate prediction of the behaviour of the PDN during transient conditions and its incorporation in system analyses for electric power transmission. This is especially critical as it can constitute the benchmark for respective decision-making processes on the stability of power systems. As a result, the core distribution networks should be constantly aware of the conditions and characteristics of each connected RES unit so that the corresponding power quality, stability and efficiency of the network are in line with the predefined requirements and specifications.

Based on the usefulness and challenges of alternative energy sources (AESs), it became evident that it is crucial for PDNs to constantly possess information of the characteristics of each individual component of the involved AESs. This will ensure the stable and robust operation of networks which require a sufficient quality of the infused energy produced by AESs. Moreover, the deployment of Smart Grids is strongly dependent on the use of new wireless communications techniques from both monitoring, control and conveying critical data from/to RES units [18, 19]. To this end, cooperative communications (CCs) can be deployed in order to allow reliable transmission of information related to the aforementioned characteristics of each RES. Specifically, by using the wireless topologies of relay systems, each RES can be equivalently represented by a relay, which can receive and transmit information. It is shown that this concept increases the potential reliability of PDNs as the decisions on coupling of certain AESs over other less suitable AESs will be made upon exploiting accurate information about the necessary component parameters.

Motivated by the above and the contribution in [20], this paper introduces a useful method that provides adequate control of the power network. Specifically, this method contributes in accounting efficiently for the determination of the criteria and characteristics that are required for adequate decision making procedures on coupling and decoupling of RES. This is realized by means of wireless cooperative systems, which constitute a core part of emerging telecommunications systems. The underlying concept of such systems is that wireless signals that convey information can be transmitted through one or more 
intermediate communication nodes. It has been shown extensively that this method increases the performance of communication links substantially because the associated impairments during wireless propagation are drastically minimized. Given also that this method practically reduces the distance between a source and a destination, the corresponding costs are minimized, whereas the probability of a system failure becomes rather low. In the present work, we adopt such a wireless deployment for representing a communication topology assuming that RES units act as source relay terminal or relay node and substations act as either source or destination nodes. Such a structure enables rapid and continuous information of the PDN on the operational characteristics and overall structure of RES units that can determine the decisions on the set-point of each RES effectively. This is performed by additionally taking into account the detrimental effects of multipath fading, which distort information signals during wireless transmission. This is implemented in the context of the outage probability (OP) performance metric, which is a critical and popular measure of performance used in both conventional and emerging wireless communication systems. The characteristics and specifications that constitute the current work an interesting engineering application are listed below.

1) Cooperative communications and wireless relaying transmission are the key features of modern wireless communications standards such as the 3rd generation partnership project long term evolution-Advanced (3GPP LTE-Advanced). Therefore, by using the benefits of cooperative relaying transmission in a WSN in dispersed RESs, the reliability and efficiency of a PDN increase significantly.

2) Due to harsh conditions in a PDN, the sensor nodes should be able to transmit all the critical data from the electrical network and provide adequate information to control stations ensuring the stability of the network. Therefore, the most significant performance metric of a WSN deployed in a PDN (i.e., a smart grid) is the nodes' outage-temporary or permanent-which is evaluated by means of the OP performance metric.

3) The direct application of prior knowledge on cooperative communications with DF relays in a dispersed RES network has not been previously introduced.

4) Closed-form expressions are derived for the OP when the channels between experience Nakagami- $m$ fading. So the engineers can easily evaluate the probability of a sensor node being in outage i.e., the probability of a RES unit experiences an outage event.

The remainder of the paper is organized as follows: Section 2 presents the considered system and channel model with detailed description of the involved components and corresponding operation. The outage probability measure and the multipath fading statistical model are introduced in Sect. 3 where simple analytic expressions for the considered scenario are derived in closed-form. The offered results are subsequently employed in Sect. 4 for evaluating extensively the performance of the considered system for various communication scenarios in both severe and moderate multipath fading conditions. Closing remarks are finally provided in Sect. 5 .

\section{System and channel models}

\subsection{PDN with RES penetration}

We assume a medium voltage (MV) radial distribution network with high RES penetration level. This is illustrated in Fig. 1, where is it shown that any MV bus of this network may have RES generation, in terms of active and reactive power, an energy backup unit (EB) and loads. Therefore, a wireless sensor node (WSN) has to be installed at each MV bus so as to gather and transfer all necessary information to the corresponding substation control unit. Based on this, the respective transmitted signals consist of the following useful data:

1) Active and reactive RES power generation.

2) Statutory information of continuous monitoring on RES unit availability.

3) EB unit state of charging.

4) Active and reactive load consumption.

5) MV bus voltage level along with the corresponding phase angle.

6) RMS current level at the point of common coupling (PCC).

7) Active power level at the PCC.

8) Reporting on any voltage disturbance incident such as overvoltage, undervoltage, voltage asymmetry, excessive harmonic distortion.

9) Reporting on any fault incident or system malfunction on both the unit and the corresponding reaction of the protection scheme.

Likewise, each MV bus may accept directives from the substation control room through its WSN with regards to the following characteristics:

1) RES generation dispatching schemes.

2) Load shedding schemes.

3) Anti-islanding directions (connect or disconnect directions).

4) Load forecast.

5) RES generation forecast. 


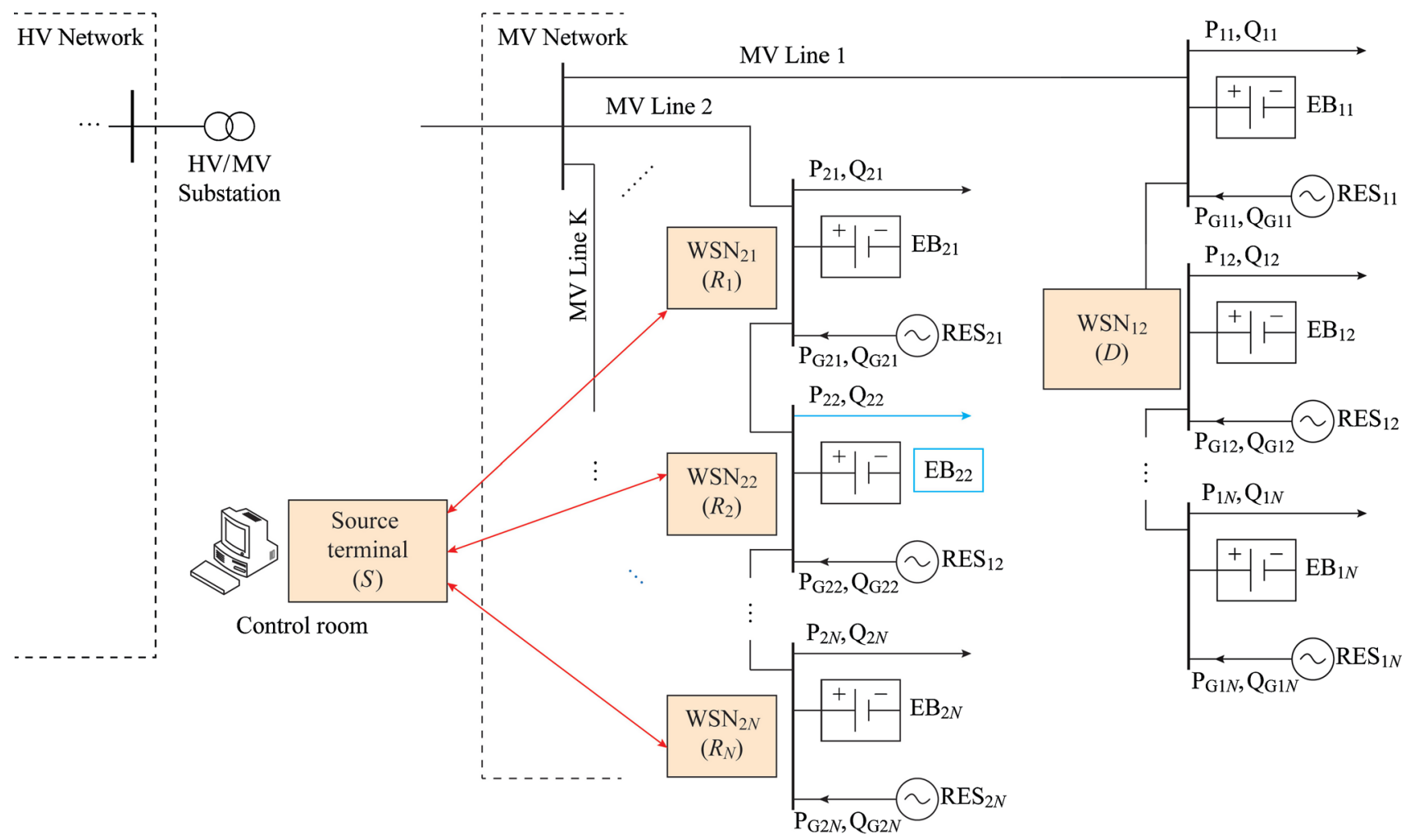

RES: Renewable Energy Source Unit; EB: Energy Backup Unit; WSN: Wireless Sensor Node

Fig. 1 A MV radial distribution network with high RES penetration with deployment of a wireless CC network

The MV radial distribution network with high RES installations can be equivalently represented as an $N$ dualhop wireless CC network which operates over multipath fading conditions that affect communication signals during wireless propagation. Such systems are widely known as cooperative diversity systems due to the use of relay nodes that receive information signals from a source node and forward them to a destination after either amplification or decoding and subsequent re-encoding. It is recalled here that CC systems are among the major emerging wireless technologies capable of providing significant advances in terms of efficiency, connectivity and quality of service. Furthermore, they have been shown to constitute a key contributor towards minimizing traditional requirements for fixed telecommunications infrastructure [21-24].

In general, cooperative networks are multi-hop communication networks where the destination-terminal combines the signals received from both source-terminal and relays [25-27]. This is a particularly critical procedure as the signal combining process aims to minimize the corresponding information errors in the presence of fading. Notably, fading is the varying distortion of wireless propagated signals which are subject to reflection, diffraction, refraction and scattering phenomena and have been shown to affect the performance and stability of wireless communication systems. Therefore, it is evident that analyzing the performance of multi-hop wireless communication networks over fading channels has been an important field of research [22-27].

\subsection{Relay system description}

In Fig. 1, a dual-hop regenerative ${ }^{1}$ relay system that consists of a source node $S$, a destination node $D$, and $N$ half-duplex relays, each of which is denoted by $R_{k}$, $k=1,2, \ldots, N$ is considered. Specifically, it is assumed that the source terminal in control room $(S)$ communicates with the destination terminal $\mathrm{WSN}_{12}(D)$ through $N$ relays (i.e. RES-nodes), $\mathrm{WSN}_{21}\left(R_{1}\right), \mathrm{WSN}_{22}\left(R_{2}\right), \ldots, \mathrm{WSN}_{2 N}$ $\left(R_{N}\right)$. In the first time slot, the source broadcasts a symbol to the relays. Then, in the second time slot, selection diversity is applied, i.e. the relay with the best end-to-end channel is selected to forward the signal to the destination while the source remains silent. It is noted here that the destination is assumed to have perfect channel state

\footnotetext{
${ }^{1}$ Regenerative cooperative systems are those that relay nodes decode the received signal and re-encode it prior to forwarding it in the destination. On the contrary, non-regenerative cooperative systems are those where relay nodes amplify and then forward the signals to the destination
} 
information, which is feasible in deployments relating to power systems. To this effect, maximum-likelihood combining of the signals from the source and the selected relay can be employed. Notably, the destination terminal (i.e. a WSN) can also act as a source node and transmit its information signals retrieved by RES to the control room (destination node) via dual-hop transmissions. Note that in Fig. 1 both scenarios were depicted where the control room and each WSN can act as either a source node or destination terminal.

The control room is equipped with a transmitting/receiving antenna and can activate/deactivate each of the WSN by transmitting a pilot signal. Therefore, if the information of a specific MV bus is needed, the control room activates the WSN assigned to this MV bus to transmit its information to the control room not only directly, but also via a number of predetermined WSNs. Each of the activated WSNs of the multi-hop communication network forwards the received information signal from its preceding node to the next one. The rest WSNs remain idle. Note that the control room is fully aware of the status of each WSN and can activate a certain number of WSNs to form an appropriate multi-hop communication network. The WSNs can gather from RES nodes all the appropriate information and play the role of intermediate regenerative relays from one hop to the next, which are able to decode-and-forward (DF) the information received by its preceding WSN. It is assumed that these WSNs can transmit over orthogonal time slots to ensure half-duplex operation and avoid any inter-signal interference. It is also assumed that each terminal $S$ transmits a signal with an average power normalized to unity.

\section{Outage probability of regenerative systems in fading conditions}

OP constitutes a vital performance measure of wireless communication systems. By recalling that wireless data rate transmission is largely a function of the respective signal noise ratio (SNR), the OP denotes the probability that the SNR level drops below a certain pre-defined threshold which results in the failure in satisfying the expected QoS requirements [28]. The outage probability is defined as follows,

$P_{\text {out }}=\int_{0}^{\gamma_{\text {th }}} p_{\gamma}(\gamma) \mathrm{d} \gamma$

where $p_{\gamma}(\gamma)$ denotes the probability density function (PDF) of the corresponding fading statistics and $\gamma_{t h}$ is the predefined SNR threshold under which transmission is suspended [28].

\subsection{Multipath fading channel}

Fading is an effect that distorts wireless information signals during wireless transmission. Its effect on the performance of conventional and emerging communication systems has been critical and thus, in depth investigations on accurate channel characterization and modelling have been extensive. In this context, Nakagami- $m$ distribution has been shown extensively to provide accurate fit in both severe and moderate multipath fading conditions. Based on this, the corresponding SNR per symbol is distributed according to the following PDF [28],

$p_{\gamma}(\gamma)=\frac{m^{m} \gamma^{m-1}}{\bar{\gamma}^{m} \Gamma(m)} \mathrm{e}^{-\frac{m \gamma}{\bar{\gamma}}}$

where $\bar{\gamma}$ and $m$ are the average SNR per symbol and the Nakagami parameter that denotes the severity of fading, respectively, whereas $\Gamma(\bullet)$ denotes the Euler gamma function [29]. Evidently, the OP of a conventional wireless communication system over Nakagami- $m$ fading channels is obtained by substituting (2) into (1). However, this does not apply readily in cooperative systems.

\subsection{OP of decode-and-forward systems over Nakagami- $m$ fading channels}

The wireless channel is also corrupted by additivewhite-Gaussian-noise (AWGN) with single-sided power spectral density $N_{0}$. The Nakagami- $m$ fading parameters in the source-relay paths (first hop) and relays-to-destination paths (second hop) are denoted as $m_{1, k}$ and $m_{2, k}$, respectively, whereas the corresponding SNRs are given by $\gamma_{1, k}$ and $\gamma_{2, k}$, respectively. Based on this, the average SNRs are given by $\bar{\gamma}_{1, k}=\Omega_{S R_{k}} E_{S} / N_{0}, \bar{\gamma}_{2, k}=\Omega_{R_{k} D} E_{S} / N_{0}$, with $\Omega_{S R_{k}}, \Omega_{R_{k} D}$, and $E_{S}$ denoting the average fading power of the first hop, the average fading power of the second hop and the energy per transmitted information symbol, respectively. To this effect, the cumulative distribution function (CDF) of the aforementioned regenerative system over Nakagami- $m$ fading can be expressed as $[23,24]$,

$F_{\gamma_{k}}\left(\gamma_{\kappa}\right)=1-\frac{\Gamma\left(m_{1, k}, \frac{m_{1, k} \gamma_{1, k}}{\bar{\gamma}_{1, k}}\right)}{\Gamma\left(m_{1, k}\right)} \frac{\Gamma\left(m_{2 k}, \frac{m_{2, k} \gamma_{2, k}}{\bar{\gamma}_{2, k}}\right)}{\Gamma\left(m_{2, k}\right)}$,

where $\Omega_{S R_{k}}=\Omega_{R_{k} D}=1$ due to normalization and

$\Gamma(a, x) \triangleq \int_{x}^{\infty} t^{a-1} \mathrm{e}^{-t} \mathrm{~d} t$

is the upper incomplete gamma function which for $x=0$ reduces to the Euler gamma function i.e. $\Gamma(a, x=0)=$ $\Gamma(a)$ [29]. By also recalling that $G(a, x) \triangleq \Gamma(a, x) / \Gamma(x)$ denotes the generalized upper incomplete gamma function, 
Eq. (3) can be equivalently re-written in the following simpler form,

$F_{\gamma_{k}}\left(\gamma_{k}\right)=1-G\left(m_{1, k}, \frac{m_{1, k} \gamma}{\Omega_{S R_{k}} \bar{\gamma}}\right) G\left(m_{2, k}, \frac{m_{2, k} \gamma}{\Omega_{R_{k}} D}\right)$.

By recalling that for every communication system over fading channels, $P_{\text {out }}=F\left(\gamma_{\text {th }}\right)$ and based on (5), the OP for the case of $N$ relays is expressed as follows,

$P_{\text {out }}=\prod_{k=1}^{N}\left\{1-G\left(m_{1, k}, \frac{m_{1, k} \gamma_{t h}}{\Omega_{S R_{k}} \bar{\gamma}}\right) G\left(m_{2, k}, \frac{m_{2, k} \gamma_{t h}}{\Omega_{R_{k}} D}\right)\right\}$.

It is noted here that the worst case fading scenario is when $m=0.5$. By also recalling that $\Gamma(1 / 2, x) \triangleq \sqrt{\pi} \operatorname{erfc}(\sqrt{x})$ it immediately follows that the OP for the worst case scenario is expressed as,

$P_{\text {out }}=\prod_{k=1}^{N}\left\{1-\pi \operatorname{erfc}\left(\sqrt{\frac{\gamma_{\text {th }}}{2 \Omega_{S R_{k}} \bar{\gamma}}}\right) \operatorname{erfc}\left(\sqrt{\frac{\gamma_{\text {th }}}{2 \Omega_{R_{k} D} \bar{\gamma}}}\right)\right\}$.

In the same context, for the special case that the value of the severity of fading parameters are positive integers i.e. $m_{1, k} \in \mathbb{N}$ and $m_{2, k} \in \mathbb{N}$, the $\Gamma(a, x)$ can be expressed in terms of a finite series given in [29, Eq. (8.352.4)]. Therefore, by performing the necessary change of variables and substituting in (6), one obtains the following closedform expression that consists only of elementary functions,

$$
\begin{aligned}
P_{\text {out }}= & \prod_{k=1}^{N}\left\{1-\mathrm{e}^{-\left(\frac{m_{1, k}}{\Omega_{S R_{k}} \gamma^{\gamma}} \frac{m_{2, k}}{\Omega_{R_{k}} D \vartheta}\right) \gamma_{\text {th }}}\right. \\
& \left.\times \sum_{i=0}^{m_{1, k}-1} \sum_{j=0}^{m_{2, k}-1} \frac{m_{1, k}^{i} m_{2, k}^{j} \gamma_{t h}^{i+j}}{i ! j ! \Omega_{S R_{k}}^{i} \Omega_{R_{k} D}^{j} \bar{\gamma}^{i+j}}\right\} .
\end{aligned}
$$

Hence, for the special case that $m_{1, k}=m_{2, k}=1$, the above expression reduces to the OP expression for Rayleigh multipath fading channels, namely,

$P_{\text {out }}=\prod_{k=1}^{N}\left\{1-\frac{\mathrm{e}^{-\left(\frac{\gamma_{\mathrm{h}}}{\Omega_{\mathrm{SR}_{\mathrm{k}}} \gamma}+\frac{\gamma_{\mathrm{th}}}{\Omega_{\mathrm{R}_{\mathrm{k}} \mathrm{D}}}\right)}}{\Omega_{S R_{k}} \Omega_{R_{k} D} \bar{\gamma}^{2}}\right\}$.

\section{Performance analysis}

This section is devoted to the analysis of the performance of OP for different communication scenarios. In more details, Fig. 2 illustrates the OP as a function of the average SNR for Rayleigh fading conditions and different values of $\gamma_{t h}$. One can observe that the OP behaviour depends highly on the value of the pre-determined threshold since even slight variations create a considerable

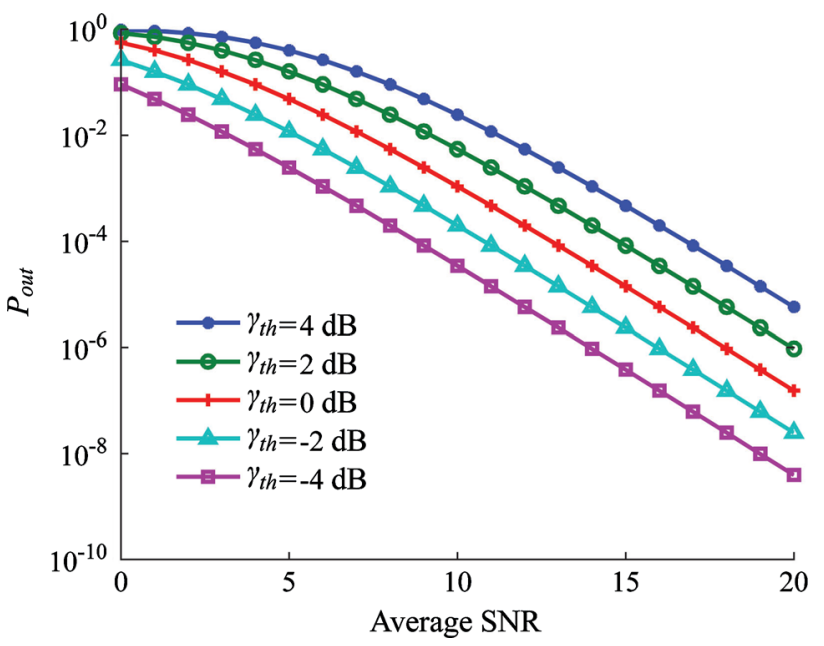

Fig. 2 OP vs $\bar{\gamma}$ for the Rayleigh case $(m=1), \Omega_{S R_{k}}=\Omega_{R_{k} D}=1.0$ and different values of $\gamma_{t h}$

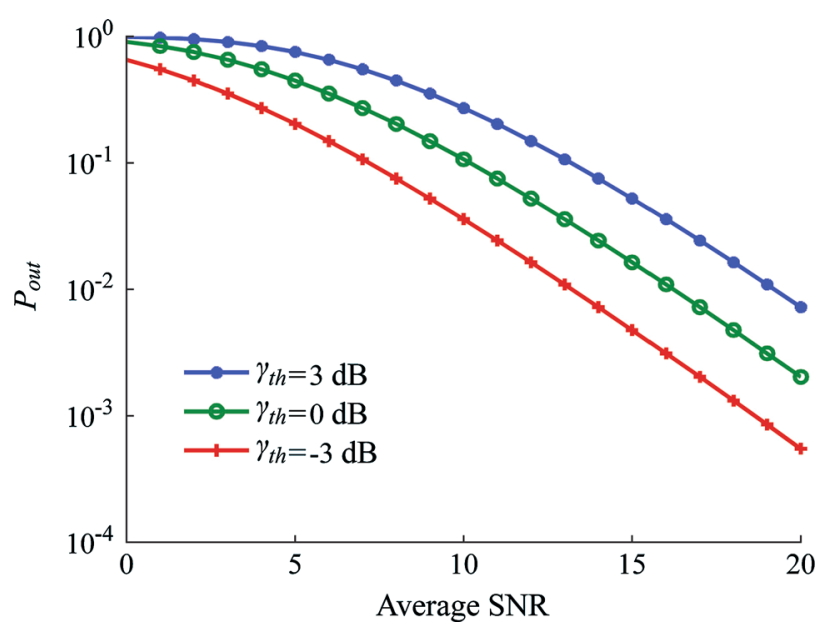

Fig. 3 OP vs $\bar{\gamma}$ for $\Omega_{S R_{k}}=\Omega_{R_{k} D}=1.0, \gamma_{t h}=-3 \mathrm{~dB}, \gamma_{t h}=0 \mathrm{~dB}$ and $m_{1, k}=m_{2, k}=0.5$

impact on the corresponding performance. This is particularly evident in the critical area of low SNR regimes where the OP lies between 1 and $10^{-3}$.

In the same context, Fig. 3 depicts the OP for three different $\gamma_{t h}$ cases for the worst case fading scenario i.e. $m_{1, k}=m_{2, k}=0.5$. Clearly, the performance of the cooperative system is rather sensitive on the severity variations of the fading conditions. For example, in comparison to the Rayleigh fading scenario considered in Fig. 3, the OP has decreased by at least an order of magnitude for comparable average SNR and threshold SNR values.

The crucial effect of fading is also demonstrated in Fig. 4. Four different scenarios are considered for quantifying the effect of fading both in terms of its severity and on different fading conditions in the pre-relay and postrelay links. The four considered scenarios are: i) 


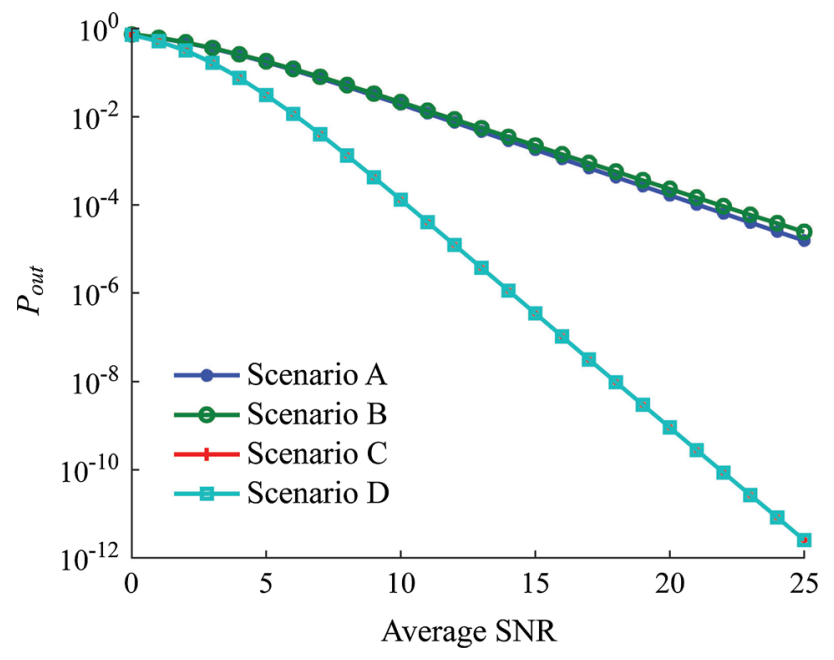

Fig. 4 OP vs $\bar{\gamma}$ for different fading conditions with $\Omega_{S R_{k}}=\Omega_{R_{k} D}=$ 1.0 and $\gamma_{\text {th }}=1 \mathrm{~dB}$

$m_{1,1}=0.5, m_{1,2}=0.5, m_{1,3}=0.5, m_{14}=0.5, m_{2,1}=0.5$, $m_{2,2}=0.8, \quad m_{2,3}=1.2, \quad m_{2,4}=2.0 ; \quad$ ii) $\quad m_{1,1}=0.5$, $m_{1,2}=0.8, \quad m_{1,3}=1.2, \quad \mathrm{~m}_{1,4}=2.0, \quad \mathrm{~m}_{2,1}=0.5$, $\mathrm{m}_{2,2}=0.4, \quad m_{2,3}=0.5, \quad m_{2,4}=0.5 ; \quad$ iii $) \quad \mathrm{m}_{1,1}=2.0$, $\mathrm{m}_{1,2}=2.0, \quad \mathrm{~m}_{1,3}=2.0, \quad m_{1,4}=2.0, \quad m_{2,1}=0.6$, $m_{2,2}=0.9, \quad m_{2,3}=1.5, \quad m_{2,4}=2.5 ; \quad$ iv) $\quad m_{1,1}=0.6$; $m_{1,2}=0.9 ; \quad m_{1,3}=1.5 ; \quad m_{1,4}=2.5 ; \quad m_{2,1}=2.0$; $m_{2,2}=2.0 ; m_{2,3}=2.0 ; m_{2,4}=2.0$. Based on this, it shows that the switching of the fading conditions between the $\mathrm{S}-\mathrm{R}$ and $\mathrm{R}-\mathrm{D}$ paths does not create a significant variation in the OP performance. Nevertheless, the effect of severity of fading is substantial in all cases as the OP varies significantly.

In the same context, the OP variation is illustrated in Fig. 5 as a function of the pre-determined threshold for different values of $\bar{\gamma}$ and the fading conditions in Scenario

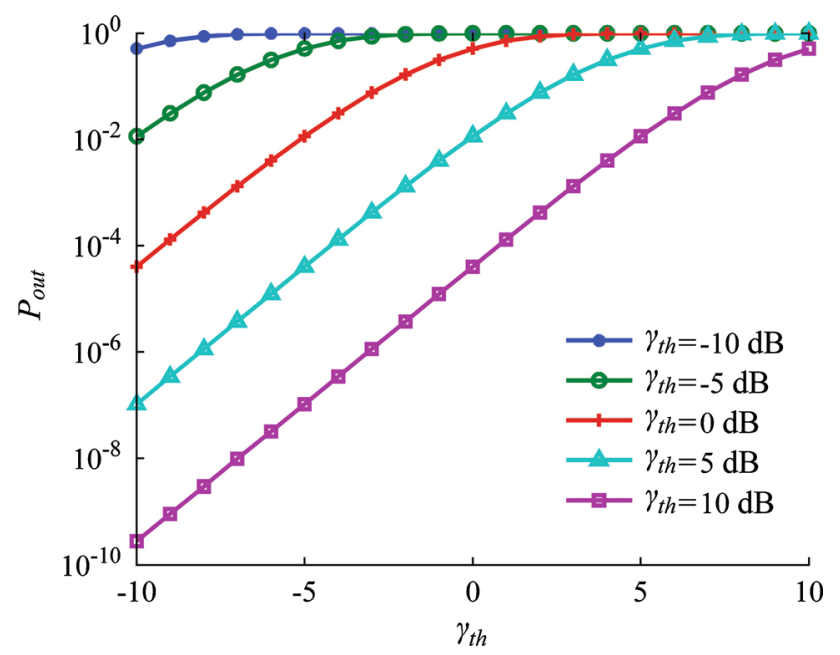

Fig. 5 OP vs $\gamma_{\text {th }}$ for different $\bar{\gamma}$ values and fixed fading according to Scenarios A, B, C and D with $\Omega_{S R_{k}}=\Omega_{R_{k} D}=1.0$
A, Scenario B, Scenario C and Scenario D, that were considered in Fig. 4. One can clearly notice that the OP increases proportionally to $\gamma_{t h}$ and its value is, as expected, unity when the $\bar{\gamma}$ is smaller than it. On the contrary, the greater the value of $\bar{\gamma}$ compared to $\gamma_{t h}$, the smaller the OP level, and thus the most robust the established communication between the involved units.

Given the nature of power system networks, it is anticipated that the offered results will be useful in future deployments subject to efficient consideration of the fading conditions that will ensure minimal OP values and thus an effective and robust system operation.

\section{Conclusion}

This paper was devoted to the deployment possibilities of CC systems in PDNs that constitute several RESs. Given the high requirements of RESs for joining the network, it is important that the characteristics of each component are fully available upon making a decision on the suitability of each RES at a given scenario. It was extensively shown that this can be achieved by employing CC concepts where relay nodes can act as RESs. To this effect, the status of each RES is fully available at the PDN with high reliability as it has been shown that the corresponding errors are significantly low. This was quantified by means of the outage probability measure which is frequently used in analyzing the performance of conventional and emerging wireless mobile communication systems. Different fading conditions were also taken into account and it was shown that thorough consideration of the corresponding effects can lead to efficient and reliable designs of future power network systems.

Acknowledgment This work was supported by the Research Program DGRES (MIS 380360) within the Research Activity ARCHIMEDES III, funded by the NSRF 2007-2013, Greece.

Open Access This article is distributed under the terms of the Creative Commons Attribution 4.0 International License (http://creativecommons.org/licenses/by/4.0/), which permits unrestricted use, distribution, and reproduction in any medium, provided you give appropriate credit to the original author(s) and the source, provide a link to the Creative Commons license, and indicate if changes were made.

\section{References}

[1] Campbell CJ, Laherrere JH (1998) The end of cheap oil. Sci Am 278:78-83

[2] Mitchell JFB (1989) The "greenhouse" effect and climate change. Rev Geophys 27:115-139

[3] Runci P, Dooley J (2004) Energy R\&D in the European Union. PNWD-3474, Pacific Northwest National Laboratory, US Department of Energy, Richland 
[4] Kurokawa K, Kamisako K, Shimizu T (1997) Conceptual considerations on PV systems composed of AC modules. Sol Energy Mater Sol Cells 47:243-250

[5] Chen Z, Guerrer JM, Blaabjerg F (2009) A review of the state of the art of power electronics for wind turbines. IEEE Trans Power Electron 24(8):1859-1875

[6] Beheraa RK, Gaob W (2010) A novel controller design for gridside converter of doubly fed induction generator for wind power interface: an experimental investigation. Electr Power Compon Syst 38(14):1531-1545

[7] Camm EH, Williams SE (2011) Solar power plant design and interconnection. In: Proceedings of the 2011 IEEE power and energy society general meeting, San Diego, 24-29 Jul 2011, 3 pp

[8] Alatrash H, Mensah A, Mark E et al (2012) Generator emulation controls for photovoltaic inverters. IEEE Trans Smart Grid 3(2):996-1011

[9] Vasquez JC, Mastromauro RA, Guerrero JM et al (2009) Voltage support provided by a droop-controlled multifunctional inverter. IEEE Trans Ind Electron 56(11):4510-4519

[10] Khederzadeh M, Maleki H (2013) frequency control of microgrids in autonomous mode by a novel control scheme based on droop characteristics. Electr Power Compon Syst 41(1):16-30

[11] El-Khattam W, Elnady A, Salama MMA (2004) Dynamic voltage restorer cost reduction in the distributed generation environment. Electr Power Compon Syst 32(6):611-626

[12] Singh B, Jayaprakash P, Kothari DP (2009) Three-leg voltage source converter integrated with $\mathrm{t}$-connected transformer as three-phase four-wire distribution static compensator for power quality improvement. Electr Power Compon Syst 37(8):817-831

[13] Venkatesh C, Siva Sarma DVSS, Sydulu M (2011) Mitigation of voltage sag/swell using peak detector based pulse width modulation switched autotransformer. Electr Power Compon Syst 39(11):1117-1133

[14] Athanasiadis N (2003) Power quality solutions for voltage sags using dynamic voltage restorers. Electr Power Compon Syst 31(2):159-170

[15] Ghamri A, Benchouia MT, Golea A (2012) Sliding-mode control based three-phase shunt active power filter: simulation and experimentation. Electr Power Compon Syst 40(4):383-398

[16] Gupta N, Singh SP, Bansal RC (2012) A digital signal processor based performance evaluation of three-phase four-wire shunt active filter for harmonic elimination, reactive power compensation, and balancing of non-linear loads under non-ideal mains voltages. Electr Power Compon Syst 40(10):1119-1148

[17] Tsang KM, Chan WL, Tang X (2013) Multi-level shunt active power filter using modular cascade H-bridge and delay firing. Electr Power Compon Syst 41(6):605-618

[18] Gungor VC, Lu B, Hancke GP (2010) Opportunities and challenges of wireless sensor networks in smart grid. IEEE Trans Ind Electron 57(10):3557-3564

[19] El Brak M, Essaaidi M (2012) Wireless sensor network in smart grid technology: challenges and opportunities. In: Proceedings of the 6th international conference on sciences of electronics, technologies of information and telecommunications (SETIT'12), Sousse, 21-24 Mar 2012, pp 578-583

[20] Tsiftsis T, Papanikolaou N, Loupis M et al (2013) On the application of cooperative communications in renewable energy sources for maximizing the reliability of power distribution networks. J Green Eng 3(4):403-420

[21] Boyer J, Falconer DD, Yanikomeroglu H (2004) Multihop diversity in wireless relaying channels. IEEE Trans Commun 52(10): 1820-1830

[22] Hasna MO, Alouini MS (2003) Outage probability of multihop transmission over Nakagami fading channels. IEEE Commun Lett 7(5):216-218
[23] Duong TQ, Quoc Bao VN, Zepernick HJ (2009) On the performance of selection decode-and-forward relay networks over Nakagami- $m$ fading channels. IEEE Commun Lett 13(3):172-174

[24] Alexandropoulos GC, Sofotasios PC, Ho-Van K et al (2013) Symbol error probability of DF relay selection over arbitrary Nakagami- $m$ fading channels. J Eng: ID 325045

[25] Sendonaris A, Erkrip E, Aazhang B (2003) User cooperation diversity, Part I: system description. IEEE Trans Commun 51(11):1927-1938

[26] Sendonaris A, Erkrip E, Aazhang B (2003) User cooperation diversity, Part II: implementation aspects and performance analysis. IEEE Trans Commun 51(11):1939-1948

[27] Laneman JN, Tse DNC, Wornell GW (2004) Cooperative diversity in wireless networks: efficient protocols and outage behaviour. IEEE Trans Inf Theory 50(12):3062-3080

[28] Simon MK, Alouini MS (2005) Digital communication over fading channels, 2nd edn. Wiley, New York

[29] Gradshteyn IS, Ryzhik M (2000) Table of integrals, series, and products, 6th edn. Academic Press, New York

Theodoros A. TSIFTSIS was born in Lamia, Greece, in 1970. He received the B.Sc. degree in physics from the Aristotle University of Thessaloniki, Greece, in 1993; the M.Sc. degree in digital systems engineering from the Heriot-Watt University, Edinburgh, U.K., in 1995; the M.Sc. degree in decision sciences from the Athens University of Economics and Business, Greece, in 2000; and the $\mathrm{Ph} . \mathrm{D}$. degree in electrical engineering from the University of Patras, Greece, in 2006. He is currently an Assistant Professor in the Department of Electrical Engineering at Technological Educational Institute of Central Greece. He is the author of more than 80 technical papers in scientific journals and international conferences. His research interests include the broad areas of cooperative communications, communication theory, wireless communications, and optical wireless communication systems. Dr. Tsiftsis acts as reviewer for several international journals and he is member of the Editorial Boards of IEEE Transactions on Communications and IEEE Communications Letters.

Paschalis C. SOFOTASIOS was born in Volos, Greece in 1978. He received the M.Eng. degree from the University of Newcastle upon Tyne, UK, in 2004, the M.Sc. degree from the University of Surrey, $\mathrm{UK}$, in 2006, and he Ph.D. degree from the University of Leeds, UK, in 2011. His Master's studies were funded by a scholarship from UKEPSRC and his Doctoral studies were sponsored by UK-EPSRC and Pace plc. He was a Post-Doctoral Researcher at the University of Leeds until August 2013 and he has been a Visiting Researcher at the University of California, Los Angeles, USA, Aristotle University of Thessaloniki, Greece and Tampere University of Technology, Finland. Since Fall 2013, he is a Post- Doctoral Research Fellow at the Department of Electronic and Communications Engineering of Tampere University of Technology and at the Wireless Communications Systems Group of Aristotle University of Thessaloniki. His research interests are in the areas of fading channel characterisation, cognitive radio, cooperative communications, optical wireless communications as well as in the theory of special functions and statistical distributions. He received a 2012 Exemplary Reviewer award by IEEE Communications Letters and the best paper award at the ICUFN '13.

Nick PAPANIKOLAOU received the Dipl. Eng. and the Ph.D. degrees in electrical engineering from the University of Patras, RionPatras, Greece, in 1998 and 2002, respectively. Prior to his academic career, he had been working for several years in the Hellenic Electric Energy Industry-involved with major European transmission and 
generation projects. He is currently an Assistant Professor in the Department of Electrical \& Computer Engineering, Democritus University of Thrace, Xanthi, Greece. His research interests include power electronics, renewable energy exploitation, distributed generation, energy saving, electric vehicles and power quality improvement. Dr. Papanikolaou is a Member of IEEE, CIGRE and of the Technical Chamber of Greece.

Michael I. LOUPIS was born in Athens in 1962. He earned a Dipl. Ing. in Electrical Engineering from the University of Thessaloniki, Greece in 1986, an M.Sc. in Microprocessor Engineering from the
University of Bradford, UK in 1987, a Dr. Ing. in Information Technology from the National Technical University of Athens (NTUA) in 1999 and an M.Sc. in Quality Assurance from the Greek Open University in 2006. His current research interests include software and modelling tools for embedded systems and design tools for renewable energy systems and energy management. Dr. Loupis is a Senior Member of IEEE, a Member of the Technical Chamber of Greece, the Greek Union of Electrical and Mechanical Engineers and the Greek Association of Computer Engineers. 